\title{
ON GENERALISED TOROIDAL-POLOIDAL SOLUTIONS OF VECTOR FIELD EQUATIONS
}

\author{
D. J. IVERS ${ }^{1}$
}

(Received 2 July 1986; revised 13 May 1988)

\begin{abstract}
The orthogonal coordinate systems $\xi^{i}(i=1,2,3)$ are determined, in which the generalised toroidal and poloidal fields, defined respectively by $\mathbf{T}\{T\}=\nabla \times$ $\left\{\boldsymbol{T} \nabla \xi^{1}\right\}$ and $\mathbf{S}\{S\}=\nabla \times \mathbf{T}\{S\}$, have the following three properties:

GP1 Decoupling of the vector Helmholtz equation: There exist linear differential operators $L_{1}$ and $L_{2}$ such that $\mathbf{H u}=\mathbf{0}$, where $\mathbf{H}$ is the vector Helmholtz operator [see equation (1)] and $\mathbf{u}=$ $\mathbf{T}\{T\}+\mathbf{S}\{S\}$, if and only if $L_{1} T=0$ and $L_{2} S=0$.

GP2 Orthogonality

GP3 Closure: $\nabla \times \mathbf{S}\{S\}$ is a $\mathbf{T}$ field.

Two choices of $\mathbf{T}$ and $\mathbf{S}$ fields are considered: type I fields with potentials $T$ and $S$, which may depend on $\xi^{1}, \xi^{2}$ and $\xi^{3}$, and type II fields with $\xi^{1}$-independent potentials. It is shown that properties GP1-GP3 only hold for type I fields in spherical and cylindrical coordinate systems, and for type II fields in azimuthal and cylindrical coordinate systems with axisymmetric and two-dimensional potentials, respectively. Analogues of GP1 for the vector wave and diffusion equations, and the Navier equation of linear elasticity, are also only true in the same four cases. Generalisations of type I and II $\mathbf{T}$ and $\mathbf{S}$ fields to arbitrary coordinate systems are indicated.
\end{abstract}

\section{Introduction}

The vector Helmholtz equation

$$
\mathbf{H u}=\left(\nabla^{2}+k^{2}\right) \mathbf{u}=\mathbf{0},
$$

${ }^{1}$ Department of Applied Mathematics, University of Sydney, N.S.W. 2006, Australia. (C) Copyright Australian Mathematical Society 1989, Serial-fee code 0334-2700/89 
where $\nabla^{2}=\nabla(\nabla \cdot)-\nabla \times(\nabla \times)$ is the vector Laplacian and $k$ is a constant, possesses solutions of the form (Lamb [9])

$$
\mathbf{u}=\nabla U+\mathbf{T}\{T\}+\mathbf{S}\{S\}
$$

where

$$
\mathbf{T}\{T\}=\nabla \times\{\mathbf{T r}\}, \quad \mathbf{S}\{S\}=\nabla \times \mathbf{T}\{S\},
$$

$\mathbf{r}$ being the radius vector from the origin $O$. Indeed, $\mathbf{u}$ satisfies the vector Helmholtz equation (1) if and only if $U, T$ and $S$ are solutions of the system

$$
\begin{gathered}
H T=\left(\nabla^{2}+k^{2}\right) T=0, \\
r \partial_{r} H U-L^{2} S=0, \quad H U+\partial_{r} r H S=0 .
\end{gathered}
$$

The notation $\nabla^{2}$ is used for both the scalar and vector Laplacians; $r=|\mathbf{r}|$, $\partial_{\tau} \equiv \partial / \partial r$; and $L^{2} \equiv \mathbf{L} \cdot \mathbf{L}$, where $\mathbf{L}=-\mathbf{r} \times \nabla$ is related to the quantum mechanical angular momentum operator.

The three terms on the right hand side of (2) are usually referred to respectively as the scaloidal, toroidal and poloidal parts of $\mathbf{u} ; U, T$ and $S$ are the corresponding potentials. In a spherical shell $V$ concentric with $O$, the fields satisfy the following extensive list of properties, collected here for reference; $f$ and $g$ denote arbitrary functions.

P1 Linearity

P2 Solenoidal property: $\nabla \cdot \mathbf{T}\{T\}=0, \quad \nabla \cdot \mathbf{S}\{S\}=0$.

P3 Spherical geometry: (i) $\mathbf{r} \cdot \mathbf{T}\{T\}=0$

(ii) $\mathbf{r} \cdot \mathbf{S}\{S\}=0 \Leftrightarrow \mathbf{S}\{S\}=\mathbf{0}$.

P4 Closure: $\nabla \times \mathbf{S}\{S\}=\mathbf{T}\left\{-\nabla^{2} S\right\}$.

P5 Commutativity:

$$
\left.\begin{array}{rc}
\text { (i) } & k \\
\text { (ii) } & \partial_{t} \\
\text { (iii) } & \nabla^{2} \\
\text { (iv) } & \mathrm{H}
\end{array}\right\}\left\{\begin{array}{c}
\nabla \\
\mathrm{T} \\
\mathrm{S}
\end{array}\right\}=\left\{\begin{array}{c}
\nabla \\
\mathrm{T} \\
\mathrm{S}
\end{array}\right\}\left\{\begin{array}{c}
k \\
\partial_{t} \\
\nabla^{2} \\
H
\end{array}\right.
$$

P6 Orthogonality: $\int_{V} \mathbf{T}\{T\} \cdot \nabla U d V=0 \quad \int_{V} \mathbf{T}\{T\} \cdot \mathbf{S}\{S\} d V=0$.

P7 Toroidal and poloidal gauge invariances: $\mathbf{T}\{T+f(r)\}=\mathbf{T}\{T\}$, $\mathbf{S}\{S+f(r)\}=\mathbf{S}\{S\}$.

P8 Scaloidal-poloidal guage invariance: If $U^{\prime}=U+\Phi$ and $S^{\prime}=$ $S+\Psi$, where $r \partial_{r} \Phi-L^{2} \Psi=0$ and $\Phi=\partial_{r} r \Psi=f(r)$, then $\nabla U+\mathbf{S}\{S\}=\nabla U^{\prime}+\mathbf{S}\left\{S^{\prime}\right\}$. 
P9 Completeness: Representation (2) is complete for $C^{1}$ vector fields; for solenoidal fields the scaloidal term is omitted.

P10 Uniqueness: The potentials of $\mathbf{u}$, which are determined from the equations

$$
\begin{gathered}
L^{2} T=-\mathbf{r} \cdot \nabla \times \mathbf{u}, \\
\nabla^{2} U=\nabla \cdot \mathbf{u}, \quad L^{2} S-r \partial_{r} U=-\mathbf{r} \cdot \mathbf{u},
\end{gathered}
$$

are only partially unique, for

$$
\mathbf{u}=\mathbf{0} \Leftrightarrow T=f(r), L^{2} S-r \partial_{r} U=0, \partial_{r} r S+U=g(r) .
$$

Consequently, the toroidal part of $\mathbf{u}$ is unique, but the scaloidal and poloidal parts of $\mathbf{u}$ are only unique up to the gauge of property P8.

Most of the above properties are obvious. The closure property $\mathrm{P} 4$ follows from curling the expression $\mathbf{S}\{S\}=-\left(\nabla^{2} S\right) \mathbf{r}+\nabla \partial_{r}(r S)$. The commutativity properties P5(iii) and (iv) are easy consequences of P4. The vanishing of a poloidal field without a radial component, P3(ii), follows from the identity

$$
\mathbf{r} \cdot \mathbf{S}\{S\}=-L^{2} S
$$

and the property of the operator $L^{2}$,

$$
L^{2} S=0 \Leftrightarrow \mathbf{T}\{S\}=\mathbf{0} \Leftrightarrow S=f(r),
$$

which is established by integrating the identity $S L^{2} S=\nabla \cdot(S \mathbf{r} \times \mathbf{T}\{S\})-$ $(\mathbf{T}\{S\})^{2}$ over $V$ and applying the divergence theorem. The difficult completeness property P9 is shown in [1] and [4]. Equation (5a) of P10 is $\mathbf{r} \cdot \nabla \times(2),(5 \mathrm{~b})$ is $\nabla \cdot(2)$ and (5c) is the radial component of (2). Two applications of (7) to (5) when $\mathbf{u}=\mathbf{0}$ completes P10.

The proof of (4) is easy: property P5(iv) implies, assuming $H \mathbf{u}=0$,

$$
\nabla\{H U\}+\mathbf{T}\{H T\}+\mathbf{S}\{H S\}=\mathbf{0},
$$

from which (4) follow by $P 10$, and the use of $P 7$ to remove the arbitrary functions $f$ and $g$ arising from P10. If $k \neq 0,(4 b)$ and (4c) can be simplified using the second gauge invariance property $\mathrm{P} 8$ to introduce the new potentials $U^{\prime}=$ $U-k^{-2} H U$ and $S^{\prime}=S-k^{-2} H S . U^{\prime}$ and $S^{\prime}$ give the same solution $\mathbf{u}$ as $U$ and $S$, but $H U^{\prime}=0$ and $H S^{\prime}=0$, since by $(4 \mathrm{~b}, \mathrm{c}) \nabla^{2} H U=0=\nabla^{2} H S$. This establishes a fundamental property of scaloidal, toroidal and poloidal fields:

P11 Decoupling of the vector Helmholtz equation: If $k \neq 0$, then $\mathbf{H u}=\mathbf{0} \Leftrightarrow H U=0, H T=0, H S=0$.

If $k=0$ the decoupling property $\mathrm{P} 11$ is no longer true: $\mathbf{r}=\nabla\left(r^{2} / 2\right)$ satisfies $\nabla^{2} \mathrm{r}=0$, but $\nabla^{2}\left(r^{2} / 2\right)=3 \neq 0$; usually additional conditions must be satisfied. 
For solenoidal fields the scaloidal potential can be taken as zero and then P11 does hold with $U=0$. If the vector Helmholtz equation contains a source term $\mathbf{f}$, so that $\mathbf{H u}=\mathbf{f}$, there exist $u, t$ and $s$ such that $\mathbf{f}=\nabla u+\mathbf{T}\{t\}+\mathbf{S}\{s\}$. Then $H U$, etc., in (8), and hence in (4) must be replaced by $H U-u$, etc.

There are analogues of the decoupling property P11 for vector field equations other than the Helmholtz equation, such as the wave and diffusion equations,

$$
\partial_{t}^{2} \mathbf{u}=c^{2} \nabla^{2} \mathbf{u}, \quad \partial_{t} \mathbf{u}=\eta \nabla^{2} \mathbf{u}
$$

and the Navier equation of linear elasticity,

$$
\partial_{t}^{2} \mathbf{u}=\alpha^{2} \nabla(\nabla \cdot \mathbf{u})-\beta^{2} \nabla \times \nabla \times \mathbf{u}
$$

in which the dominant term is the vector Laplacian or either of the related operators, $\nabla \times \nabla \times$ or $\nabla(\nabla \cdot)$. Equations (9) reduce to (1) if the time dependence is separated out. Alternatively, the commutativity of $\partial_{t}$ and $H$, property P5(ii), can be used to decouple (9) into scalar wave and diffusion equations for $U, T$ and $S$. Of course, decoupling will only be effective if the boundary conditions decouple. Even if complete decoupling of the potential equations cannot be achieved due to the presence of operators which do not commute with $\nabla, \mathbf{T}$ or $\mathbf{S}$, a scaloidal-toroidal-poloidal decomposition can still be useful, particularly numerically. For example, the equation

$$
\partial_{t} \mathbf{B}=\eta \nabla^{2} \mathbf{B}+\nabla \times(\mathbf{v} \times \mathbf{B}),
$$

for the solenoidal magnetic induction $\mathbf{B}$ in an electrically conducting fluid moving with velocity $\mathbf{v}$, can be decomposed into an infinite coupled set of differential equations in $t$ and $r$ with the aid of a toroidal-poloidal decomposition of $\mathbf{B}$ and spherical harmonic expansions of the potentials [3]. For freely-decaying magnetic fields, that is, when $\mathbf{v}=\mathbf{0}$, the induction equation (11) reduces to (9b).

In generalising the representation (2) and (3) to nonspherical geometries, only solenoidal fields need be considered, since scaloidal fields are defined independently of the geometry, unlike toroidal and poloidal fields, which satisfy property P3. Accordingly,

$$
\nabla \cdot \mathbf{u}=\mathbf{0} .
$$

A natural generalisation of (2) and (3) to other geometries is

$$
\mathbf{T}\{T\}=\nabla \times\left\{T \mathbf{e}^{1}\right\}, \quad \mathbf{S}\{S\}=\nabla \times \mathbf{T}\{S\},
$$

where $\mathrm{e}^{1}=\nabla \xi^{1}=\hat{\mathbf{e}}_{1} h_{1}^{-1}, \hat{\mathbf{e}}_{i}$ and $h_{i}$ being the unit vectors and scale factors of an orthogonal coordinate system $\xi^{i}(i=1,2,3)$. For example, $\nabla \times\{T \hat{\mathbf{z}}\}$ and $\nabla \times \nabla \times\{T \hat{\mathbf{z}}\}$, where $z$ is a cartesian coordinate, are commonly used in plane geometries. The fields defined by (13) will be referred to as $\mathbf{T}$ and $\mathbf{S}$ fields. Some of the properties $\mathrm{P} 1-\mathrm{P} 11$ of toroidal and poloidal fields generalise immediately, 
such as the geometrical property $\mathrm{P} 3(\mathrm{i})$ : $\mathrm{T}$ fields are orthogonal to $\xi^{1}$-surfaces, that is,

$$
\hat{\mathbf{e}}_{1} \cdot \mathbf{T}\{T\}=0,
$$

which follows from $\nabla \times \mathbf{e}^{1}=0$. The other properties are not quite as easily or obviously generalised. One generalisation of the decoupling property P11 is:

GP0 $\quad \mathbf{T}$ and $\mathbf{S}$ decoupling of the vector Helmholtz equation: There exists a function $w\left(\xi^{1}\right)$ such that $\mathrm{HT}\{T\}=0 \Leftrightarrow H\{w T\}=0$ and $\mathrm{HS}\{S\}=\mathbf{0} \Leftrightarrow H\{w S\}=0$.

It is shown in [12] that GP0 holds if the scale factors $h_{i}$ and $w$ satisfy

$$
\partial_{1} \partial_{1} w=0, \quad h_{1}=1, \quad \partial_{1}\left(h_{2} / h_{3}\right)=0,
$$

where $\partial_{1} \equiv \partial / \partial \xi^{1}$, etc. The separable coordinate systems of the scalar Helmholtz equation ([11], [12]) which satisfy conditions (15) are listed in [12]. The Navier equation (see [10]) analogue of GP0 is also true for general fields, if conditions (15) are satisfied; similar results hold for axisymmetric fields ([6]).

An alternative generalisation of toroidal and poloidal fields,

$$
\mathbf{U}_{t}=\hat{\mathbf{e}}_{1} \times \nabla T, \quad \mathbf{U}_{p}=\hat{\mathbf{e}}_{1} S+\nabla U,
$$

has been given by Rädler [13], based on a nonstandard definition of a poloidal field, $\mathbf{U}_{p}=\hat{\mathbf{r}} S+\nabla U$, which combines the more usual definitions (2) and (3) of scaloidal and poloidal fields. Unlike $\mathbf{T}$ fields, $\mathbf{U}_{t}$ fields are not solenoidal in a coordinate system unless

$$
h_{1}=h_{1}\left(\xi^{1}\right)
$$

in which case $\mathbf{U}_{t}=\mathbf{T}\left\{h_{1} T\right\}$. Rädler did not consider any generalised decoupling property for the fields $\mathbf{U}_{t}$ and $\mathbf{U}_{p}$, but did show that if

$$
\partial_{1}\left(h_{2} / h_{3}\right)=0
$$

and (17a) are satisfied, then the closure property

$$
\nabla \times \mathbf{U}_{t} \text { is a } \mathbf{U}_{p} \text { field, } \nabla \times \mathbf{U}_{p} \text { is a } \mathbf{U}_{t} \text { field, }
$$

holds. Property (18a) is not generally true, unlike the corresponding property (13b) for $T$ and $S$ fields. Condition (17a) can be reduced to (15b) by transforming to the new coordinate system $\varsigma^{i}$ given by

$$
\varsigma^{1}=\int h_{1}\left(\xi^{1}\right) d \xi^{1}, \quad \varsigma^{2}=\xi^{2}, \quad \varsigma^{3}=\xi^{3},
$$

since $U_{t}$ and $U_{p}$ are unchanged by the transformation. From the vanishing of the Riemann curvature tensor of the orthogonal metric satisfying (17), Rädler showed that the Gaussian curvature of the $\xi^{1}$-surfaces must either be zero or a positive constant. It follows from Minding's Theorem [15] that the surfaces are either planes or spheres. Rädler concluded that the coordinate system must be 
either cylindrical with $\hat{\mathbf{e}}_{1}=\hat{\mathbf{z}}$, or spherical with $\hat{\mathbf{e}}_{1}=\hat{\mathbf{r}}$, but omitted detailed justification. Some care is needed for there are other coordinate systems possessing plane coordinate surfaces, e.g. the surfaces of the azimuthal angle about an axis.

The purpose of this paper is to determine those $\mathbf{T}$ and $\mathbf{S}$ fields for which the following generalised decoupling property holds:

$\mathbf{T}$ and $\mathbf{S}$ decoupling of the vector Helmholtz equation: There are

GP1

linear scalar differential operators $L_{1}$ and $L_{2}$ such that if $\mathrm{u}$ is given by the representation (2) and (13),

$$
\mathbf{H u}=\mathbf{0} \Leftrightarrow L_{1} T=0, L_{2} S=0 .
$$

Property GP1 generalises P11 but unlike GP0 it does not require that $T$ and $S$ satisfy the same equation. Furthermore, all orthogonal coordinate systems are examined, not just separable systems of the scalar Helmholtz equation as in [12]. The results are applicable to analogues of the decoupling property GP1 for several related field equations, which will therefore not receive separate treatment (but see Section 5 for a discussion).

In Section 2, necessary conditions on $\hat{\mathbf{e}}_{i}$ and $h_{i}$ for the decoupling property GP1 to hold are derived for two types of $\mathbf{T}$ and $\mathbf{S}$ fields:

Type I: $T$ and $S$ may depend on $\xi^{1}, \xi^{2}$ and $\xi^{3}$;

Type II: the $\hat{\mathbf{e}}_{i}$-components of $\mathbf{T}$ and $\mathbf{S}$ fields are independent of $\xi^{1}$.

Type II fields generalise axisymmetric solenoidal fields. The conditions necessary for GP1 are also shown to be necessary for

GP2 Orthogonality: $\int_{V} \mathbf{T}\{T\} \cdot \mathbf{S}\{S\} d V=0$;

GP3 Closure: $\nabla \times \mathbf{S}$ is a $\mathbf{T}$ field.

The type I conditions are precisely (17).

In Section 3, $\hat{\mathbf{e}}_{i}$ and $h_{i}$ are explicitly determined for type I fields by using conditions (17) to integrate the structural equations (33) for the unit vectors $\hat{\mathbf{e}}_{i}$ and their integrability conditions (34) and (35), which are equivalent to the vanishing of the Riemann curvature tensor. The approach is more direct and complete than in [13]. The same method is used for type II fields in Section 4. Section 5 summarises the results of Sections 3 and 4, discusses analogues of GP1 for several other field equations and indicates possible generalisations of $\mathbf{T}$ and $\mathbf{S}$ fields.

\section{The conditions determining $\hat{e}_{1}$ and $\xi^{1}$}

If $T$ and $S$ are the solutions of the decoupled system of homogeneous partial differential equations (19), then $T\{T\}$ and $S\{S\}$ must individually satisfy the 
vector Helmholtz equation (1). It suffices a posteriori to examine $\mathbf{T}\{T\}$ alone, and to assume that all functions which occur are sufficiently differentiable. The following three expressions are needed:

$$
\begin{gathered}
\mathbf{T}\{T\}=\mathbf{e}^{2} H_{2} \partial_{3} T-\mathbf{e}^{3} H_{3} \partial_{2} T \\
\mathbf{S}\{S\}=-\mathbf{e}^{1} h_{1}^{2} L^{2} S+\mathbf{e}^{2} H_{2} \partial_{1} H_{3} \partial_{2} S+\mathbf{e}^{3} H_{3} \partial_{1} H_{2} \partial_{3} S
\end{gathered}
$$

and

$$
\nabla \times \mathbf{S}\{S\}=\mathbf{T}\left\{-h_{1}^{2} L^{2} S\right\}-\mathbf{e}^{2} H_{2} \partial_{1} H_{3} \partial_{1} H_{2} \partial_{3} S+\mathbf{e}^{3} H_{3} \partial_{1} H_{2} \partial_{1} H_{3} \partial_{2} S-\mathbf{e}^{1} H_{1} \Delta S,
$$

where $H_{i}=h_{i}^{2} /\left(h_{1} h_{2} h_{3}\right), L^{2} \equiv \mathrm{L} \cdot \mathbf{L}$ with $\mathrm{L}=-\mathbf{e}^{1} \times \nabla$ and

$$
\Delta S=\partial_{3} H_{2} \partial_{1} H_{3} \partial_{2} S-\partial_{2} H_{3} \partial_{1} H_{2} \partial_{3} S \text {. }
$$

If $\mathbf{T}\{T\}$ is substituted into the vector Helmholtz equation (1), and (22) is used, there results

$\mathbf{T}\left\{\left(h_{1}^{2} L^{2}+k^{2}\right) T\right\}+\mathbf{e}^{2} H_{2} \partial_{1} H_{3} \partial_{1} H_{2} \partial_{3} T-\mathbf{e}^{3} H_{3} \partial_{1} H_{2} \partial_{1} H_{3} \partial_{2} T+\mathbf{e}^{1} H_{1} \Delta T=0$.

The $\hat{\mathbf{e}}_{1}$-component of (24) is $\Delta T=0$, or

$$
\begin{aligned}
\left(H_{2} \partial_{1} H_{3}-H_{3} \partial_{1} H_{2}\right) \partial_{2} \partial_{3} T & +\left(\partial_{3} h_{1}^{-2}\right) \partial_{1} \partial_{2} T-\left(\partial_{2} h_{1}^{-2}\right) \partial_{1} \partial_{3} T \\
& +\left(\partial_{3} H_{2} \partial_{1} H_{3}\right) \partial_{2} T-\left(\partial_{2} H_{3} \partial_{1} H_{2}\right) \partial_{3} T=0
\end{aligned}
$$

expanding the operator $\Delta$ defined by (23). Comparing (20) and (24) it is clear that the second and third terms on the left-hand side of (24) constitute a $\mathbf{T}$ field if and only if there exists a potential $\tilde{T}$ such that

$$
\partial_{2} \tilde{T}=\partial_{1} H_{2} \partial_{1} H_{3} \partial_{2} T, \quad \partial_{3} \tilde{T}=\partial_{1} H_{3} \partial_{1} H_{2} \partial_{3} T .
$$

$\tilde{T}$ exists if and only if (26) are integrable, which is true if and only if $\partial_{1} \Delta T=0$; by (25) $\tilde{T}$ does exist. Equation (24) can be written as $\mathbf{T}\left\{\tilde{T}+\left(h_{1}^{2} L^{2}+k^{2}\right) T\right\}=\mathbf{0}$. Hence

$$
\tilde{T}+\left(h_{1}^{2} L^{2}+k^{2}\right) T=f\left(\xi^{1}\right),
$$

where $f$ is an arbitrary function.

If $T$ is to be determined by a single scalar equation, either (25) or (27) must be satisfied identically in $T$ through constraints on the scale factors $h_{i}$. This is impossible for (27), as an examination of the coefficients of the highest derivatives of $T$ will reveal. Thus (25) must be the identity in $T$ : for type I fields the coefficient of each derivative of $T$ in (25) must vanish. Some simple manipulations reduce the resulting equations to (17), the type I necessary conditions for property GP1.

The derivatives of type II $\mathbf{T}$ potentials are not all arbitrary for the $\hat{\mathbf{e}}_{\boldsymbol{i}}$-components of $\mathbf{T}\{T\}$ are independent of $\xi^{1}$ if and only if

$$
\partial_{1} \partial_{2} T=0, \quad \partial_{1} \partial_{3} T=0
$$


and

$$
\partial_{1}\left(h_{1} h_{2}\right)=0, \quad \partial_{1}\left(h_{1} h_{3}\right)=0 .
$$

Since an additive function of $\xi^{1}$ in the potentials $T$ and $S$ can be dropped by the obvious generalisation of property P7 to $\mathbf{T}$ and $\mathbf{S}$ fields, (28) imply type II fields have $\xi^{1}$-independent potentials. Thus the second and third terms in (25) drop out; and (25) is an identity in $T$ only if the coefficients of the remaining derivatives of $T$ vanish, that is,

$$
\partial_{2} \partial_{1} h_{1}^{-2}=0, \quad \partial_{3} \partial_{1} h_{1}^{-2}=0,
$$

noting $(29 a, b)$. Thus type II fields exist only in coordinate systems which satisfy $(29 \mathrm{a}, \mathrm{b})$, and they have the generalised decoupling property GP1 only if conditions (29) are satisfied.

$\mathbf{T}$ and $\mathbf{S}$ fields also have the orthogonality property GP2 only if (25) is satisfied. For, if $V$ is the domain of interest some standard vector manipulations yield

$$
\int_{V} \mathbf{T}\{T\} \cdot \mathbf{S}\{S\} d V=\int_{\partial V} T \mathbf{e}^{1} \times \mathbf{S}\{S\} \cdot d \mathbf{S}-\int_{V} T \mathbf{e}^{1} \cdot \nabla \times \mathbf{S}\{S\} d V,
$$

which vanishes only if

$$
\mathbf{e}^{1} \cdot \nabla \times \mathbf{S}\{S\}=0
$$

since $T$ is arbitrary or $\xi^{1}$-independent in $V$, and if $T \mathbf{e}^{1} \times \mathbf{S}\{S\} \cdot \mathbf{n}=0$ on $\partial V$ where $\mathbf{n}$ is the outward normal to $\partial V$. By (22), (30) is equivalent to (25). The second condition is satisfied without the need for any boundary conditions on $T$ or $S$ if $\mathbf{n}=\hat{\mathbf{e}}_{1}$, i.e. if $\partial V$ consists of $\xi^{1}$-surfaces; otherwise boundary conditions must be imposed on $T$ and $S$. Condition (30), and hence (25), is also necessary, and even sufficient, for $\mathbf{T}$ and $\mathbf{S}$ fields to possess the closure property GP3. For, if $\nabla \times \mathbf{S}\{S\}$ is a $\mathbf{T}$ field, (30) follows from the geometric property (14) of T fields. Conversely, if (30) holds then $\Delta S=0$, so there exists $\tilde{S}$ such that $\nabla \times \mathbf{S}\{S\}=\mathbf{T}\left\{-\tilde{S}-h_{1}^{2} L^{2} S\right\}$. Thus type I [type II] fields have the orthogonality property GP2 or the closure property GP3 only if the conditions (17) [(29)] are satisfied.

\section{Type I T and $\mathbf{S}$ fields}

From the conditions on the $h_{i}$ necessary for type I fields to have property GP1, namely (17), both $\hat{\mathbf{e}}_{1}$ and $\xi^{1}$, and hence $\mathbf{T}\{T\}$ and $\mathbf{S}\{S\}$, are determined. For this purpose are needed $(i, j, k=1,2,3)$ : the equations defining $\hat{\mathbf{e}}_{i}$ and $h_{i}$

$$
\partial_{i} \mathbf{r}=h_{i} \hat{\mathbf{e}}_{i}
$$

the orthogonality condition

$$
\hat{\mathbf{e}}_{i} \cdot \hat{\mathbf{e}}_{j}=\delta_{i j}
$$


the structural equations

$$
\partial_{i} \hat{\mathbf{e}}_{j}=\left(\partial_{j} h_{i} / h_{j}\right) \hat{\mathbf{e}}_{i}-\delta_{i j} \sum_{m=1}^{3}\left(\partial_{m} h_{j} / h_{m}\right) \hat{\mathbf{e}}_{m} ;
$$

and the integrability conditions [7] on (33), namely

$$
\partial_{i}\left(\partial_{i} h_{j} / h_{i}\right)+\partial_{j}\left(\partial_{j} h_{i} / h_{j}\right)+\left(\partial_{k} h_{i}\right)\left(\partial_{k} h_{j}\right) / h_{k}^{2}=0
$$

and

$$
\partial_{i} \partial_{j} h_{k}-\left(\partial_{j} h_{i}\right)\left(\partial_{i} h_{k}\right) / h_{i}-\left(\partial_{i} h_{j}\right)\left(\partial_{j} h_{k}\right) / h_{j}=0
$$

$\delta_{i j}$ denotes the Kronecker delta and $i \neq j \neq k \neq i$ in (34) and (35). Integrating (35) for $k=2,3$ using (17b) gives

$$
\partial_{1} h_{2} / h_{1} h_{2}=G\left(\xi^{1}\right)=\partial_{1} h_{3} / h_{1} h_{3},
$$

where $G$ is arbitrary. The cases $G \neq 0$ and $G=0$ must be treated separately.

(a) $G \neq 0$. Equations (17a), (34) for $i=1, j=2$ and (36a) give $\partial_{1} G^{-1}=h_{1}$. Thus equation (36) becomes

$$
\partial_{1} h_{2} /\left(h_{1} h_{2}\right)=1 / h=\partial_{1} h_{3} /\left(h_{1} h_{3}\right)
$$

where

$$
h=\int h_{1}\left(\xi^{1}\right) d \xi^{1} .
$$

The determination of $\hat{\mathbf{e}}_{1}$ and $\xi^{1}$ is now easy. By (31) for $i=1,(33)$ for $i=j=1$ and $(38), \partial_{1}\left(\mathbf{r}-h \hat{\mathbf{e}}_{1}\right)=0$. Further, by (31) for $i=1,(33)$ for $i=2, j=1$ and $(37), \partial_{2}\left(\mathbf{r}-h \hat{\mathbf{e}}_{1}\right)=\mathbf{0}$. Similarly, $\partial_{3}\left(\mathbf{r}-h \hat{\mathbf{e}}_{1}\right)=\mathbf{0}$. Thus $\mathbf{r}-h \hat{\mathbf{e}}_{1}$ is a constant vector, which may be taken as zero by a shift of the origin 0 . Hence the coordinate system is spherical, i.e. $\mathbf{r}=h \hat{\mathbf{e}}_{1}, \hat{\mathbf{e}}_{1}=\hat{\mathbf{r}}, h\left(\xi^{1}\right)=r$. The coordinate $\xi^{1}$ is determined up to the unknown function $h$, which remains arbitrary. $T\{T\}$ and $\mathbf{S}\{S\}$ are the usual toroidal and poloidal fields.

(b) $G=0$. From (36), $\partial_{1} h_{2}=0=\partial_{1} h_{3}$. Together with (33) for $i=1,2,3$, $j=1$ and $(17 \mathrm{a})$, this implies that $\hat{\mathbf{e}}_{1}$ is a constant vector. Then $\partial_{1}\left(\mathbf{r}-h \hat{\mathbf{e}}_{1}\right)=0$ using (31) for $i=1$ and (38). Further, by (31) for $i=2$ and (32),

$$
\partial_{2}\left[\hat{\mathbf{e}}_{1} \cdot\left(\mathbf{r}-h \hat{\mathbf{e}}_{1}\right)\right]=\hat{\mathbf{e}}_{1} \cdot \partial_{2}\left(\mathbf{r}-h \hat{\mathbf{e}}_{1}\right)=\hat{\mathbf{e}}_{1} \cdot h_{2} \hat{\mathbf{e}}_{2}=0 \text {. }
$$

Similarly, $\partial_{3}\left[\hat{\mathbf{e}}_{1} \cdot\left(\mathbf{r}-h \hat{\mathbf{e}}_{1}\right)\right]=0$. Thus $\hat{\mathbf{e}}_{1} \cdot\left(\mathbf{r}-h \hat{\mathbf{e}}_{1}\right)$ is a constant which may be set to zero by adjusting the constant of integration in $h$. Then $\mathbf{r}=h \hat{\mathbf{e}}_{1}+\mathbf{f}_{\perp}$, where $f_{\perp} \perp \hat{e}_{1}$ and $f_{\perp}$ depends only on $\xi^{2}$ and $\xi^{3}$. The coordinate system must be cylindrical and we can take $\hat{\mathbf{e}}_{1}=\hat{\mathbf{z}}$ and $h\left(\xi^{1}\right)=z$.

\section{Type II T and S Fields}

Now consider type II fields. By the conditions $(29 \mathrm{a}, \mathrm{b})$ on the $h_{i}$ for type II fields, $\partial_{1}\left(h_{2} / h_{3}\right)=0$, so that (36) holds for type II fields. [The derivation of (36) 
depended only on (17b) and (34).] Hence from (36) and (29a,b), $\partial_{1} h_{1}^{-1}=G\left(\xi^{1}\right)$. Integrating, $h_{1}^{-1}=\int G\left(\xi^{1}\right) d \xi^{1}+H\left(\xi^{2} \xi^{3}\right)$, where $H$ is arbitrary. Substituting this expression for $h_{1}^{-1}$ into (29c,d) yields $G \partial_{2} H=0=G \partial_{3} H$. Thus without loss of generality $G=0$ or $H=0$. The latter condition gives $h_{1}=h_{1}\left(\xi^{1}\right)$, which along with $\partial_{1}\left(h_{2} / h_{3}\right)=0$ has already been considered in Section 3 . Spherical coordinate systems do not satisfy conditions $\left(29_{a}, b\right)$, but cylindrical coordinates do. The new case to consider is $G=0$, which with $(29 \mathrm{a}, \mathrm{b})$ implies

$$
h_{i}=h_{i}\left(\xi^{2}, \xi^{3}\right), \quad i=1,2,3 .
$$

We now find $\hat{\mathbf{e}}_{1}$ and $\xi^{1}$ under conditions (39). By (33) for $i=2,3, j=1 \hat{\mathbf{e}}_{1}$ is a function only of $\xi^{1}$. Differentiate (33) for $i=j=1$ with respect to $\xi^{1}$ and substitute for $\partial_{1} \hat{\mathbf{e}}_{2}$ and $\partial_{1} \hat{\mathbf{e}}_{3}$ from (33) for $i=1, j=2,3$. This gives

$$
\partial_{1} \partial_{1} \hat{\mathbf{e}}_{1}+\omega^{2} \hat{\mathbf{e}}_{1}=\mathbf{0}
$$

where $\omega^{2}=\left(\partial_{2} h_{1} / h_{2}\right)^{2}+\left(\partial_{3} h_{1} / h_{3}\right)^{2}$. Equations (34) for $i=2, j=3$ and (39) imply $\partial_{2}\left(\partial_{2} h_{1} / h_{2}\right)=-\left(\partial_{3} h_{2} / h_{3}\right)\left(\partial_{3} h_{1} / h_{3}\right)$, and (35) for $i=2, j=3$ can be written as $\partial_{2}\left(\partial_{3} h_{1} / h_{3}\right)=\left(\partial_{3} h_{2} / h_{3}\right)\left(\partial_{2} h_{1} / h_{2}\right)$. Thus

$$
\partial_{2} \omega^{2} / 2=\left(\partial_{2} h_{1} / h_{2}\right) \partial_{2}\left(\partial_{2} h_{1} / h_{2}\right)+\left(\partial_{3} h_{1} / h_{3}\right) \partial_{2}\left(\partial_{3} h_{1} / h_{3}\right)=0 .
$$

Similarly, $\partial_{3} \omega^{2}=0$. Thus $\omega$ is a constant. The cases $\omega \neq 0$ and $\omega=0$ must be treated separately.

(a) $\omega \neq 0$. The general solution of $(40)$ is

$$
\hat{\mathbf{e}}_{1}=\hat{\mathbf{a}} \cos \omega \xi^{1}+\hat{\mathbf{b}} \sin \omega \xi^{1},
$$

where $\hat{\mathbf{a}}$ and $\hat{\mathbf{b}}$ are constant unit orthogonal vectors, since $\hat{\mathbf{e}}_{1}$ is a unit vector. Subsituting (41) into (31) for $i=1$ and integrating gives

$$
\mathbf{r}=h_{1}\left(\xi^{2}, \xi^{3}\right)\left(\hat{\mathbf{a}} \sin \omega \xi^{1}-\hat{\mathbf{b}} \cos \omega \xi^{1}\right) \omega^{-1}+\mathbf{g}\left(\xi^{2}, \xi^{3}\right),
$$

where $\mathrm{g}$ is arbitrary. Now, by (42) and (31) for $i=2$,

$$
\partial_{2}(\hat{\mathbf{a}} \cdot \mathbf{g})=\hat{\mathbf{a}} \cdot h_{2} \hat{\mathbf{e}}_{2}-\left(\partial_{2} h_{1}\right) \omega^{-1} \sin \omega \xi^{1} .
$$

But substituting (41) into $\hat{\mathbf{e}}_{2} \cdot(33)$ for $i=j=1$ yields

$$
\partial_{2} h_{1} / h_{2}=\hat{\mathbf{e}}_{2} \cdot \hat{\mathbf{a}} \omega \sin \omega \xi^{1}-\hat{\mathbf{e}}_{2} \cdot \hat{\mathbf{b}} \omega \cos \omega \xi^{1} .
$$

Moreover, $0=\hat{\mathbf{e}}_{2} \cdot \hat{\mathbf{e}}_{1}=\hat{\mathbf{e}}_{2} \cdot \hat{\mathbf{a}} \cos \omega \xi^{1}+\hat{\mathbf{e}}_{2} \cdot \hat{\mathbf{b}} \sin \omega \xi^{1}$. Hence, from the last two equations, $h_{1} \hat{\mathbf{e}}_{2} \cdot \hat{\mathbf{a}}=\left(\partial_{2} h_{1}\right) \omega^{-1} \sin \omega \xi^{1}$, so $\partial_{2}(\hat{\mathbf{a}} \cdot \mathbf{g})=0$. Similarly, $\partial_{3}(\hat{\mathbf{a}} \cdot \mathbf{g})=$ $0=\partial_{2}(\hat{\mathbf{b}} \cdot \mathbf{g})=\partial_{3}(\hat{\mathbf{b}} \cdot \mathbf{g})$. Thus $\hat{\mathbf{a}} \cdot \mathbf{g}$ and $\hat{\mathbf{b}} \cdot \mathbf{g}$ are constants, which can be adjusted to zero by a shift of the origin 0 . Introducing cartesian coordinates $(x, y, z)$ and choosing the appropriate sign for $\omega$ we obtain $\mathbf{r}=h_{1}\left(\xi^{2}, \xi^{3}\right) \omega^{-1}$ $\left(\hat{\mathbf{x}} \cos \omega \xi^{1}+\hat{\mathbf{y}} \sin \omega \xi^{1}\right)+z\left(\xi^{2}, \xi^{3}\right) \hat{\mathbf{z}}$. Accordingly the coordinate system must be azimuthal: indeed, we can choose $\hat{\mathbf{e}}_{1}=\hat{\phi}$ and $\xi^{1}=\phi \omega^{-1}$, where $(s, \phi, z)$ are cylindrical polar coordinates. The associated $\mathbf{T}$ and $\mathbf{S}$ fields are axisymmetric 
and provide a complete representation of axisymmetric solenoidal fields; $T$ is essentially Stokes' stream function. The $\mathbf{S}$ field has the simple form $\mathbf{S}\{S\}=s A \hat{\phi}$, where $A=-s^{-2}\left(\nabla^{2}-2 s^{-1} \partial_{s}\right) S$ is independent of $\phi$.

(b) $\omega=0$. Then $\partial_{2} h_{1}=0=\partial_{3} h_{1}$, and this is a special case of $\mathrm{I}$ (b) (see Section 3), where $h_{1}$ is a constant and $T$ is independent of $z$. The coordinate system is cylindrical and the $\mathbf{T}$ and $\mathbf{S}$ fields are two-dimensional. Analogously to the previous case $\mathrm{II}(\mathrm{a}), \mathbf{S}\{S\}=A \hat{\mathbf{z}}$, where $A=-\nabla^{2} S$ is independent of $z$.

\section{Summary and concluding remarks}

The following table summarises the results of Sections 3 and 4 .

\begin{tabular}{lccccc} 
Type & Description & $\xi^{1}$ & $\hat{\mathbf{e}}_{1}$ & $L_{1} T$ & $L_{2} S$ \\
\hline I(a) & spherical & $r$ & $\hat{\mathbf{r}}$ & $H\left(r^{-1} T\right)$ & $H\left(r^{-1} S\right)$ \\
I(b) & cylindrical & $z$ & $\hat{\mathbf{z}}$ & $H T$ & $H S$ \\
II(a) & azimuthal/axisymmetric & $\phi$ & $s^{-1} \hat{\phi}$ & $\left(H-2 s^{-1} \partial_{s}\right) T$ & $\left(H+2 s^{-1} \partial_{s}\right) A$ \\
II(b) & cylindrical/two-dimensional & $z$ & $\hat{\mathbf{z}}$ & $H T$ & $H A$
\end{tabular}

The simplifications of type II(a) and II(b) fields from Section 4 have been used, namely

$$
\begin{aligned}
& \mathrm{II}(\mathrm{a}) \quad A=-s^{-2}\left(\nabla^{2}-2 s^{-1} \partial_{s}\right) S, \quad \mathrm{~S}\{S\}=s A \hat{\phi} \text {; } \\
& \text { II(b) } \quad A=-\nabla^{2} S, \quad \mathbf{S}\{S\}=A \hat{\mathbf{z}} \text {. }
\end{aligned}
$$

A type II $\mathbf{T}$ field or $\mathbf{S}$ field can be expressed respectively as a type I $\mathbf{S}$ field or $\mathbf{T}$ field with an appropriate potential, but the type II form with $A$ rather than $S$ is generally simpler.

The $\xi^{1}$-surfaces are concentric spheres in I(a), parallel planes in I(b) and II(b), and meridional planes in II(a). The spherical or planar nature of $\xi^{1}$-surfaces is a result of $(17 \mathrm{~b})$, namely $\partial_{1}\left(h_{2} / h_{3}\right)=0$ (which is not made clear in [13]), and Minding's Theorem, for the Gaussian curvature [15] of the $\xi^{1}$-surfaces in an orthogonal coordinate system is $K=-1 /\left(h_{2} h_{3}\right)\left[\partial_{2}\left(\partial_{2} h_{3} / h_{2}\right)+\partial_{3}\left(\partial_{3} h_{2} / h_{3}\right)\right]$, which simplifies to a nonnegative constant

$$
K=\left(\partial_{1} h_{2} /\left(h_{1} h_{2}\right)\right)\left(\partial_{1} h_{3} /\left(h_{1} h_{3}\right)\right)=\left[G\left(\xi^{1}\right)\right]^{2}
$$

using (34) for $i=2, j=3$ and then (36).

The only $\mathbf{T}$ and $\mathbf{S}$ fields possessing the orthogonality property GP2 are the four listed above. Type I(a) spherical $\mathbf{T}$ and $\mathbf{S}$ fields are orthogonal over a spherical shell concentric with 0 (see Section 1). Type I(b) cylindrical $\mathbf{T}$ and $\mathbf{S}$ fields are orthogonal over an infinite slab, say $a<z<b$, if the potentials $T$ and $S$ decay sufficiently rapidly as $s \rightarrow \infty$. The two type II fields have 
the stronger algebraic orthogonality property: $\mathbf{T}\{T\} \cdot \mathbf{S}\{S\}=0$ for all $\xi^{1}$ independent potentials $T$ and $S$. The table also gives the only four $\mathbf{T}$ and $\mathbf{S}$ fields, which have the closure property GP3.

The decoupling of the $\mathbf{T}$ and $\mathbf{S}$ potential equations is actually a property of the vector Laplacian $\nabla^{2}$ or of the operator $\nabla \times \nabla \times$ since the fields are solenoidal and $k^{2}$ commutes with $\mathbf{T}$ and $\mathbf{S}$. Analogues of the decoupling property GP1 for other field equations, whose operators are the vector Laplacian plus operators which commute with $\mathbf{T}$ and $\mathbf{S}$, hold only in the same four cases. In particular, this is true for the vector wave and diffusion equations, and the Navier equation of linear elasticity. $\mathbf{T}$ and $\mathbf{S}$ decoupling of the magnetic induction equation is restricted to the above four cases at most. Complete decoupling only occurs in special cases that depend on $\mathbf{v}$ through the term $\nabla \times(\mathbf{v} \times \mathbf{B})$, but they usually lead to antidynamo theorems such as the axisymmetric theorem (Cowling [5], [8]), in which the magnetic induction field $\mathbf{B}$ cannot be maintained against ohmic losses by the inductive effects of $\mathbf{v}$; that is, self-exciting dynamo action cannot occur.

There are two ways in which the results of this paper might possibly be extended: by allowing more general $\mathbf{T}$ and $\mathbf{S}$ fields and by considering more general properties of such fields. The definition (13) of $\mathbf{T}$ and $\mathbf{S}$ fields can be generalised by prescribing the basis field without any reference to any coordinate system $\xi^{i}$. Under this generalisation the geometrical condition (14), $\hat{\mathbf{e}}_{\mathbf{1}} \cdot \mathbf{T}\{T\}=$ 0 , can be retained if and only if

$$
\mathbf{e}^{1} \cdot \nabla \times \mathbf{e}^{1}=0
$$

This apparent relaxation of the condition $\nabla \times \mathbf{e}^{1}=\mathbf{0}$, which any coordinate basis field satisfies, is illusory, for (43) implies $\mathrm{e}^{1}=\alpha \nabla \beta$ for some $\alpha$ and $\beta$, and $\nabla \beta$ clearly generates the same $\mathbf{T}$ and $\mathbf{S}$ fields as $\mathbf{e}^{1}$. Thus the most general choice for $\mathbf{e}^{1}$, if the geometrical condition (14) is retained, is essentially $\mathbf{e}^{1}=\nabla \xi^{1}$ for some function $\xi^{1}=\xi^{1}(x, y, z)$. A curvilinear coordinate system $\xi^{i}$ containing $\xi^{1}$ can always be constructed if $\nabla \xi^{1} \neq 0$; but it can be orthogonal if and only if $\xi^{1}$ is a solution of a particular third order partial differential equation [7]. Now a coordinate system $\xi^{i}$, unless it is orthogonal, possesses two distinct canonical sets of basic vectors: the tangent vectors along the coordinate curves, $\mathbf{e}_{i}=\partial_{2} \mathbf{r}$, and the normals to the coordinate surfaces, $\mathbf{e}^{i}=\nabla \xi^{i}$. The two sets $\mathbf{e}^{i}$ and $\mathbf{e}_{j}$ are reciprocal, that is, $\mathbf{e}^{i} \cdot \mathbf{e}_{j}=\delta_{j}^{i}, \mathbf{e}^{i}=J^{-1} \mathbf{e}_{j} \times \mathbf{e}_{k}, \mathbf{e}_{i}=J \mathbf{e}^{j} \times \mathbf{e}^{k}$, where $(i, j, k)$ is a cyclic permutation of $(1,2,3)$ and $J=\mathbf{e}_{1} \cdot \mathbf{e}_{2} \times \mathbf{e}_{3}=\left(\mathbf{e}^{1} \cdot \mathbf{e}^{2} \times \mathbf{e}^{3}\right)^{-1}$ is the Jacobian, $\left|\partial x^{i} / \partial \xi^{j}\right|$, of the transformation from the cartesian coordinates $x^{i}$ to $\xi^{\imath}$. Beside the $\mathbf{T}$ and $\mathbf{S}$ fields defined by (13) using the basis field $\mathbf{e}^{1}$ another class of $\mathbf{T}$ and $\mathbf{S}$ fields can be naturally associated with a coordinate system $\boldsymbol{\xi}^{i}$ using the basis field $\mathbf{e}_{1}$,

$$
\mathbf{T}\{T\}=J^{-1} \mathbf{e}_{1} \times \nabla T, \quad \mathbf{S}\{S\}=J^{-1} \mathbf{e}_{1} S,
$$


where $T$ and $S$ are $\xi^{1}$-independent. [Definition (13) is not appropriate for $\mathbf{e}_{1}$ since $\nabla \times e_{1}$ is not necessarily zero.] The $T$ field (44a) is solenoidal if and only if $\left(\nabla \times J^{-1} \mathbf{e}_{1}\right) \cdot \nabla T=0$ for all $\xi^{1}$-independent $T$, i.e. if and only if $\mathbf{e}_{1} \times \nabla \times J^{-1} \mathbf{e}_{1}=$ 0 . The $S$ field (44b) is solenoidal since $\nabla \cdot\left(J^{-1} \mathbf{e}_{1}\right)=0$. [Note that the gradient operator $\nabla=\sum_{i} \mathbf{e}^{i} \partial_{i}$, so that $\mathbf{e}_{1} \cdot \nabla \equiv \partial_{1}$.] The $\mathbf{T}$ and $\mathbf{S}$ fields (44) are the correct generalisation of type II fields to an arbitrary coordinate system. One could replace (44) by $\mathbf{T}\{T\}=\mathbf{w} \times \nabla T, \mathbf{S}\{S\}=\mathbf{w} S$, where $\mathbf{w} \cdot \nabla T=0=\mathbf{w} \cdot \nabla S$, $\nabla \cdot \mathbf{w}=\mathbf{0}$ and $\mathbf{w} \times \nabla \times \mathbf{w}=\mathbf{0}$, so that the fields are solenoidal. However, if $w \neq 0$ a coordinate system $\xi^{i}$ can be found [2] such that $w=e_{1}$ and $w \cdot \nabla \equiv \partial_{1}$.

The second possible extension is to allow more general properties. For example complete decoupling of the potential equations could be relaxed to various degrees of coupling. The difficulty with this is the greater complexity of coupled systems of partial differential equations. One successful analytical example is that of Lortz [10], who has used the type II fields of the coordinate system

$$
\xi^{1}=-k s^{2} \phi+l z, \quad \xi^{2}=k^{2} \ln \left(l^{2}+k^{2} s^{2}\right) / 2, \quad \xi^{3}=l \phi+k z,
$$

where $k$ and $l$ are integers and $k l \neq 0$, to construct self-exciting helical solutions of the magnetic induction equation (11) from a coupled system of potential equations. The properties of the type I $T$ and $S$ fields (13) and the type II fields (44) in an arbitrary coordinate system will be the subject of future work.

In some circumstances the solenoidal condition (12) can be imposed on solutions of (1) or other field equations as an initial or boundary condition; an example of the latter for (11) in a cylindrical geometry is given in [14]. This approach to condition (12) is sometimes preferable to the use of a solenoidal representation of the solution, particularly when the boundary conditions would couple the $\mathbf{T}$ and $\mathbf{S}$ fields.

\section{References}

[1] G. E. Backus, "A class of self-sustaining dissipative spherical dynamos", Ann. Phys, 4 (1958) 372-447.

[2] R. L. Bishop and S. I. Goldberg, Tensor analysis of manifolds (Dover Publications, New York, 1980).

[3] E. C. Bullard and H. Gellman, "Homogeneous dynamos and terrestrial magnetism", Phil. Trans. Roy. Soc. Lond. A 247 (1954) 213-278.

[4] P. Chadwick and E. A. Trowbridge, "Elastic wave fields generated by scalar wave functions", Proc. Camb. Phil. Soc. 63 (1967) 1177-1187.

[5] T. G. Cowling, "The magnetic field of sunspots", Mon. Not. Roy. Astron. Soc. 94 (1934) $39-48$.

[6] A. C. Eringen and E. S. Suhubi, Elastodynamics Vol. 2, Linear theory (Academic Press, New York, 1975).

[7] A. R. Forsyth, Differential geometry (Cambridge University Press, Cambridge, 1912). 
[8] D. J. Ivers and R. W. James, "Axisymmetric antidynamo theorems in compressible nonuniform conducting fluids", Phil. Trans. Roy. Soc. Lond. A 312 (1984) 179-218.

[9] H. Lamb, "On the oscillations of a viscous spheroid", Proc. Lond. Math. Soc. 13 (1881), $51-66$.

[10] D. Lortz, "Exact solutions of the hydromagnetic dynamo problem", Plasma Phys. 10 (1968) 967-972.

[11] P. Moon and D. E. Spencer, Field theory for engineers (Van Nostrand, Princeton, 1961).

[12] P. M. Morse and H. Feshbach, Methods of theoretical physics, Part II (McGraw-Hill Book Company, New York, 1953).

[13] K. H. Rädler, "Zur dynamotheorie kosmischer magnetfelder. II. Darstellung von vektorfeldern als summe aus einem poloidalen und einem toroidalen anteil", Astron. Nachr. 295 (1974) 73-84.

[14] S. I. Vainshtein, "Simplest dynamo instability", Sov. Phys. J.E.T.P. 41 (1976) 494-497.

[15] T. J. Willmore, An introduction to differential geometry (Oxford University Press, Oxford, 1959). 